\title{
On the Performance of Lossless Wavelet Compression Scheme on Digital Medical Images in JPEG, PNG, BMP and TIFF Formats
}

\author{
Richard O. Oyeleke \\ Department of Computer \\ Sciences, University of Lagos, \\ Nigeria
}

\author{
Femi O. Alamu \\ Department of Computer \\ Science \& Information \\ Technology, Bowen University, \\ Nigeria
}

\author{
Agnes Akinwole \\ Department of Computer \\ Science, Yaba College of \\ Technology, Nigeria
}

\begin{abstract}
The major challenges facing digital radiological systems notably: teleradiology and picture archiving and communication systems (PACS) are not unconnected to: limited storage space and bandwidth requirements for storage, transmission, retrieval and archiving of digital medical images. Lossless compression techniques have been adopted by the medical community to help solve these problems as it does not degrades the quality of the medical image which is crucial for diagnosis while reducing the size (often in ratio 2:1) of the original image. However, little attention has been paid to knowing the performance of a choice compression scheme on the medical image format type for efficient handling in the digital radiology system. Hence, this work presents an evaluation of the performance of a lossless wavelet compression scheme on samples of computed tomography (CT) images of the brain in JPEG, PNG, BMP and TIFF formats.
\end{abstract}

\section{General Terms}

Image Processing, Health Informatics

\section{Keywords}

Wavelet compression, Medical images, Image formats, Compression ratio

\section{INTRODUCTION}

The field of radiology has enjoyed a major progress with its driver being the advances in digital technology. It has paved way for newer approaches to rendering diagnostic services. [7]. Computed tomography (CT), magnetic resonance imaging (MRI), and ultrasonography (US) to mention a few, have been crucial in acquisition of clearer and quality images of internal structure of human body which otherwise would not have been possible with conventional radiograph [3].

Digital medical images open up a whole new range to acquisition, processing, storage, transportation, accessing and retrieval of images. It paved way for the development and adoption of systems like; teleradiology and picture archiving and communication systems (PACS), which have helped both patients and medical personnel to overcome the barriers of distance and access to limited or scarce expertise for diagnosis [1]. Despite these benefits, digital medical images appear not be handful as they usually are generated in tons of gigabyte, hence, require a great deal of resources: especially network and space requirements faster transmission and efficient storage. Since digital radiological systems aimed at improving radiodiagnostic services, timely transmission of medical images from a remote radiology centre to another is crucial to patient's survival [3].
One major technique that has been employed by the medical community to resolving these issues is through "compression" (i.e. removal of redundancy in image data thereby reducing the original size of the image). Many compression algorithms exist but little attention have been paid to their performance on images saved in different formats. Since digital images are made up of picture elements with varying amount and intensity on the different types of image formats that exist, compression ratio yielded by an algorithm can depend greatly on the level of orderliness and the amount of redundancy of the image data [10]. Therefore, this work examines the compression performance of lossless wavelet compression on selected image formats viz: JPEG, PNG, BMP and TIFF formats.

\section{DIGITAL MEDICAL IMAGES}

Digital images are made up of picture elements simply referred to as 'pixels', each pixel characterized by its unique color values [2]. Medical images acquired with newer imaging modalities such as CT and MRI have aided physicians in carrying out painless and more accurate diagnosis of patient's health condition. They offer better view of the internal structure of the human body thereby allowing easier location of the troubled area of the human body [5]. More so, they are more portable to handle than the conventional radiographs thereby offering simultaneous view by physicians at remote locations, better storage and transportation compared to film-based images [1]. Digital images, without doubt, have helped in the increasing use of teleradiology in expanding healthcare coverage beyond the four walls of hospitals, affording remote areas to access and benefit from quality healthcare services.

\section{IMAGE FORMATS}

Digital images can exist in different formats, but more commonly used formats are the: joint photographic expert group (JPEG /JPG), portable network graphics (PNG), bitmap (BMP) and TIFF formats.

\subsection{Portable Network Graphics (PNG)}

The PNG format was developed primarily to substitute both the GIF and TIFF formats. It is based on lossless compression, hence, the process of saving or viewing the image will cause no loss of image data [9]. The PNG is saved with 256 colors maximum but it saves the color information more efficiently. It also supports an 8 bit transparency PNG can support 24 bit RGB color images, grayscale images, both with and without alpha channels [4]. 


\subsection{Joint Photographic Expert Group (JPEG / JPG)}

JPEG is the most common image format used on the web, as it offers a very high compression ratio [11]. JPEG makes use of a lossy compression scheme, hence, images suffer degradation in quality as a result of loss of information each time it is saved. It is no surprise that JPEG file size is almost always smaller than a PNG, especially for photographic images [9]

\subsection{Bitmap (BMP)}

BMP files are image files that show the way windows stores bit mapped images [8]. Bitmap files are usually large and uncompressed, but the images are rich in color, of high quality, simple and compatible in all windows OS and programs. These files are made of millions and millions of dots called ,pixelse, with different colors and arrangements to come up with an image or pattern. It might an 8-bit, 16-bit or 24-bit image [4].

\subsection{Tagged Image File Format (TIFF/ TIF)}

TIFF format is very flexible and dynamic especially in terms of image storage. Multiple images can be stored in a single file unlike other image formats [9]. It comes in both lossy or lossless forms and it is supported by several imaging programs. It is capable of recording halftone image data with different pixel intensities, thus is the perfect format for graphic storage, processing and printing [4].

\section{COMPRESSION TECHNIQUES}

Compression is the technique that has been widely embraced as way to solving the problem of storage and bandwidth requirement for image transmission and archiving. Compression simply, is the removal of redundancy in image data in order to achieve significant reduction in size for shorter transmission time, faster transmission rate and of course, reduced storage requirement. Basically, there exist two forms of compression techniques, namely; lossless and lossy compression [6].

\subsection{Lossless Compression}

This scheme compresses image data with a no loss of information, although the image size is reduced, usually offering a compression ratio of around $2: 1$ to $8: 1$ but the quality is not degraded [10]. As image quality is very crucial for accurate diagnosis, lossless schemes are used in compressing digital medical images.

\subsection{Lossy Compression}

This scheme offers great potential with significant reduction in the size of the original image than the lossless scheme counterpart, however, there is loss of image data and the quality is compromised [3]. Despite offering a better solution for storage and bandwidth requirement for image transmission, it is prone to diagnostic errors which may endanger the life of patients.

\section{IMPLEMENTATION}

For this work, we employed a lossless wavelet compression scheme using MATLAB version R2012a image processing tool kit. Wavelet compression is a form of data compression which is well suited for image compression. Its primary aim is to store image data in as little space as possible in a file. We applied this wavelet compression on samples of digital image (specifically a CT scan image of the brain) in the following formats: JPEG, PNG, BMP and TIFF as shown in Fig 1 below. The images all have same dimension $(434 \times 556)$ but their sizes vary as measured in kilobytes (KB).

First, once the wavelet compression scheme is applied, the image is subjected to a transform which produces as many coefficients as there are pixels in the image. This allows for an easier compression of the coefficients produced because the information is statistically concentrated in just a few coefficients. This process is referred to as transform coding. Finally, the quantized values are entropy encoded [12]. 


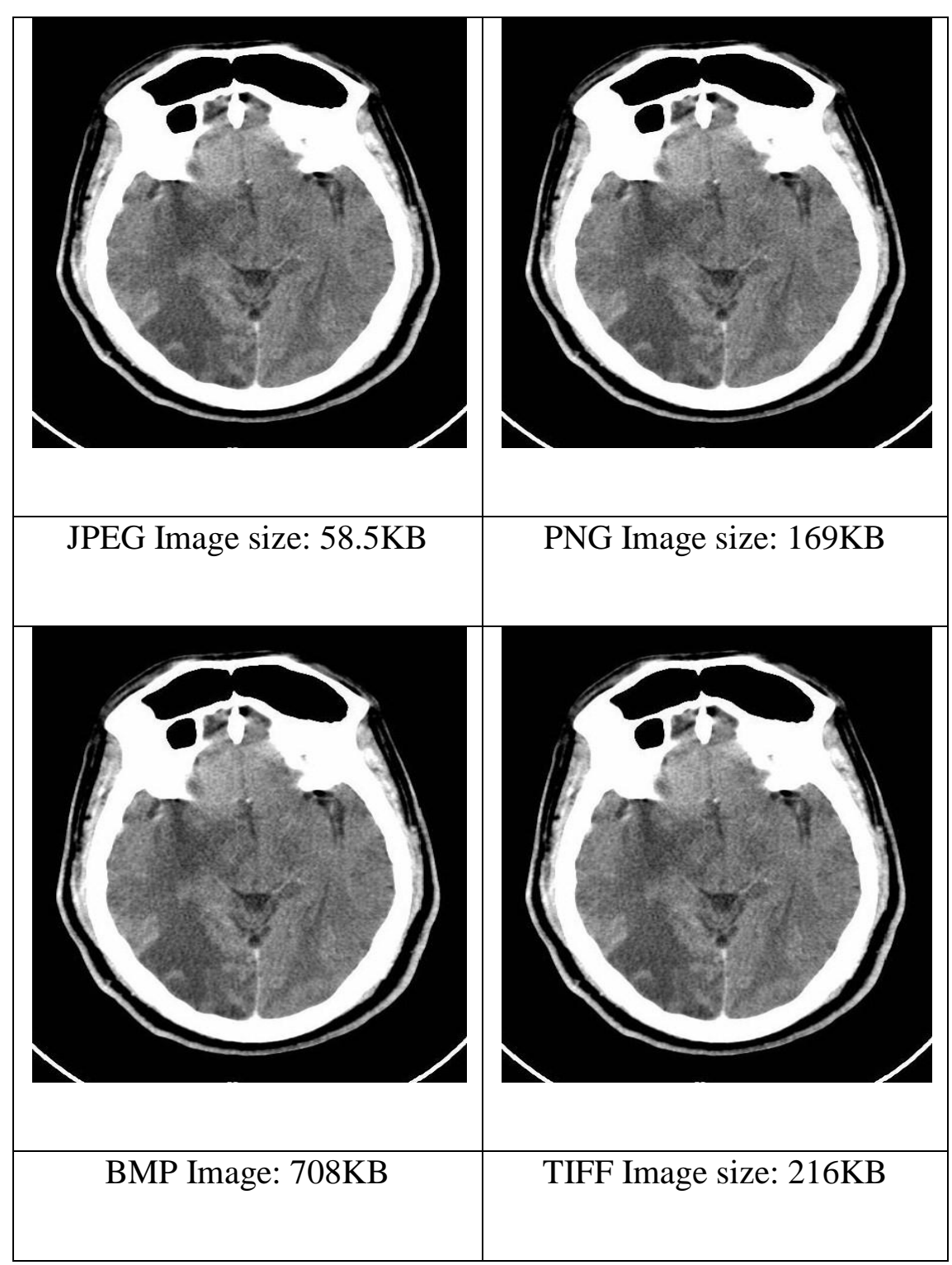

Fig 1: CT Images of a brain infarction before compression 


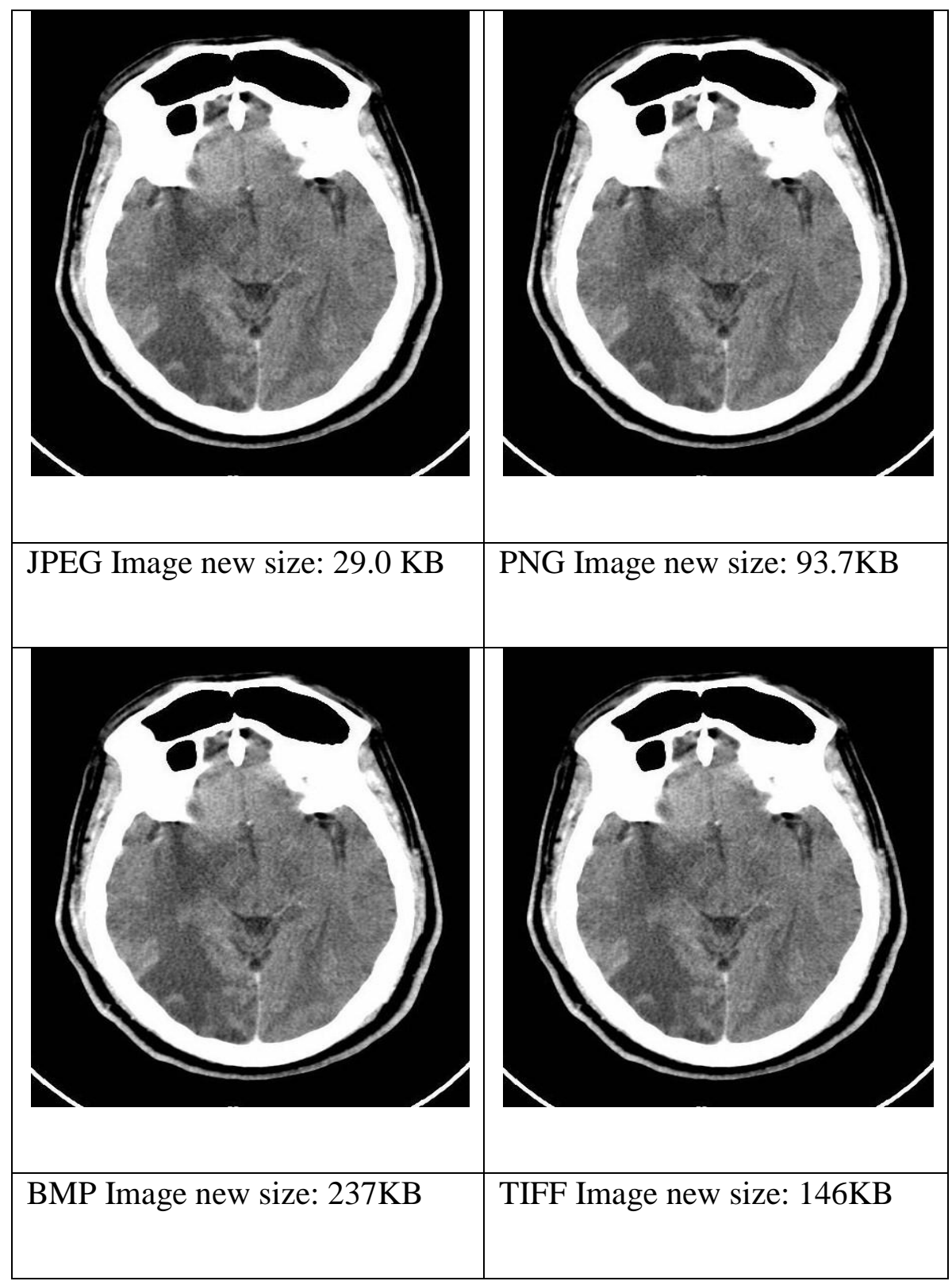

Fig 2: CT Images of a brain infarction before compression

\section{RESULTS}

Fig 2 above shows the outputs of the compression of each of the CT images formats. It was observed that the dimensions of each of the image formats remain the same $(434 \times 556)$ as they were before compression. More so, none of the image formats suffered degradation in quality, but their sizes were further reduced.

Table 1 summarizes the performance of the lossless wavelet compression of the CT images of the brain in the different formats. From Table 1, the BMP image format ranked 1st which implied that the lossless wavelet compression scheme had its best performance on this format. The compressed BMP image had a size $237 \mathrm{~KB}$ as against its original size of $708 \mathrm{~KB}$, which approximated to a ratio of about $3: 1$. Next to this performance, was on the JPEG format, as it returned an image size of $29.0 \mathrm{~KB}$ after compression as against its original size of $58.8 \mathrm{~KB}$, hence, ranked $2 \mathrm{nd}$. The performance on PNG image format ranked 3rd, as it returned a compressed image size of 93.7KB as against its initial size of $169 \mathrm{~KB}$ which gave a close approximate compression ratio of $2: 1$, although not as good as the performance on JPEG image format. Finally, the lossless wavelet scheme performance on TIFF image format was ranked 4th (i.e. reported the least performance) as it gave a compression size of $146 \mathrm{~KB}$ as against the original size of 216KB. Not much compression was achieved, as the compression ratio approximates to $1: 1$. 
Table 1. Performance of lossless wavelet compression Scheme on digital medical images in JPEG, PNG, BMP and TIFF formats

\begin{tabular}{|c|c|c|c|c|}
\hline Image Formats & Dimension & $\begin{array}{c}\text { Image Size Before } \\
\text { Compression (KB) }\end{array}$ & $\begin{array}{c}\text { Image Size After } \\
\text { Compression (KB) }\end{array}$ & Ranking \\
\hline BMP Image & $434 \times 556$ & 708 & 237 & $2 \mathrm{nd}$ \\
\hline JPEG Image & $434 \times 556$ & 58.5 & 29.0 & $3 \mathrm{rd}$ \\
\hline PNG Image & $434 \times 556$ & 169 & 93.7 & 4 th \\
\hline TIFF Image & $434 \times 556$ & 216 & 146 & \\
\hline
\end{tabular}

\section{CONCLUSION}

Tons of image data are generated on a daily basis in radiodiagnosis centres on a daily basis, with the limited space and bandwidths posing threats to digital radiology systems such as; PACS and teleradiology, there is need to finding alternatives to help overcome these challenges. Lossless compression has been adopted by the medical professionals as it does not degrade the image quality which is crucial for accurate diagnosis. However, little concern is given to knowing; "what compression scheme is better for what image format?" from our results, lossless wavelet compression performed best on the CT image of the brain in bitmap (BMP) format. Next was on JPEG format, which was closely followed by the PNG format and had the least performance on TIFF format.

\section{REFERENCES}

[1] De Backer AI, Mortelé KJ, De Keulenae BL. Picture archiving and communication system - Part 1. Filmless Radiology and Distance Radiology. 2004; 87: 234-241.

[2] Deserno TM, editor. Fundamentals of biomedical image processing. Springer-Verlag: Berlin Heildeberg; 2011.

[3] Kumar Y.P. and Mishra K. (2006).Term Paper On; A survey of Contemporary Medical Compression Techniques, Department of Electronics and Communication Engineering Guwahati

[4] Rachel, A. (2011, January). Different Image Formats And When to Use Them. Retrieved February 15, 2013, from

1stwebdesigner: http://www.1stwebdesigner.com/design/different-imageformats/

[5] Rao K.M.M. and Rao V.D.P. (2006). Medical Image Processing

[6] Sridevi S, Vijayakuymar V.R, and Anuja R. (2012). A Survey on Various Compression Methods for Medical Images, I.J. Intelligent Systems and Applications, 2012, 3, 13-19.

[7] Vidar Systems Corporation (2010). The Transition To Digital Imaging In Medicine: 1 other coverage criteria for logical decisions", Journal of The Integral Role of Film Digitizers

[8] Wouters W. (2008). BMP Format: Windows Bitmap File Format Specifications, Clean Coding Company.

[9] url: http://www.atalasoft.com/png

[10] Al-laham M. and El Emary I. M. M.(2007). Comparative Study Between Various Algorithms of Data Compression Techniques, Proceedings of the World Congress on Engineering and Computer Science, San Francisco, USA.

[11] Wayne, F. (2010). Image file formats - TIF, JPG, PNG, GIF. Retrieved February 15, 2013, from SCANTIPS: http://www.scantips.com/basics09.html

[12] url: en.wikipedia.org/wiki/Wavelet_transform 